\title{
COMPARAÇÃO DE DIFERENTES ABORDAGENS PARA AVALIAÇÃO DA INCERTEZA NA CROMATOGRAFIA GASOSA DO GÁS NATURAL
}

\author{
Elcio Cruz de Oliveira* \\ PETROBRAS TRANSPORTE S.A. - Gerência Geral de Engenharia, Av. Presidente Vargas, 328, 20091-060 Rio de Janeiro - RJ, Brasil \\ Paula Fernandes de Aguiar \\ Departamento de Química Analítica, Instituto de Química, Universidade Federal do Rio de Janeiro, Cidade Universitária - Ilha \\ do Fundão, CT, Bloco A, 21949-900 Rio de Janeiro - RJ, Brasil
}

Recebido em 8/10/08; aceito em 12/2/09; publicado na web em 3/7/09

\begin{abstract}
COMPARISON OF DIFFERENT APPROACHES TO EVALUATE THE UNCERTAINTY OF GAS CHROMATOGRAPHY FOR NATURAL GAS. The evaluation of uncertainty associated with an analytic result is an essential part of the measurement process. Recently, several approaches to evaluate the uncertainty in measurement have been developed. Here, the gas chromatography assay uncertainty for natural gas is compared by some of these approaches: the guide to the expression of uncertainty in measurement (GUM) approach, top-down approach (reproducibility estimate from an inter-laboratory study), Barwick \& Ellison (data from validation), study of variability and fuzzy approach. The comparison shows that GUM, Barwick \& Ellison and fuzzy approaches lead to comparable uncertainty evaluations, which does not happen with the top-down approach and study of variability by the absence of data normality.
\end{abstract}

Keywords: measurement uncertainty; GUM approach; top-down approach.

\section{INTRODUÇÃO}

A incerteza de uma medição é definida como "um parâmetro, associado ao resultado de uma medição, que caracteriza a dispersão dos valores que podem ser fundamentalmente atribuídos a um mensurando". ${ }^{1}$ O resultado de uma medição é considerado como a melhor estimativa do valor do mensurando acompanhado de todas as fontes da incerteza que contribuem à propagação do mesmo. ${ }^{2,3}$ Consequentemente, um resultado de medição não pode corretamente ser interpretado sem conhecimento sobre a incerteza deste resultado. ${ }^{2}$

Vários conceitos foram desenvolvidos para a avaliação de incerteza relacionada a um resultado de medição. Uma das primeiras abordagens para avaliar a incerteza em Química Analítica foi publicada nos anos 80. Wernimont usou estimativas de precisão a partir de estudos interlaboratoriais em desempenho de métodos para avaliar a incerteza. ${ }^{4}$ Outra abordagem para a expressão da incerteza de medição é proposta pelo GUM. ${ }^{3}$ Esta abordagem combina as diversas fontes de incerteza, através da expansão da série de Taylor. No início dos anos 1990, o EURACHEM adotou o GUM como referência de uso em Química Analítica. ${ }^{5}$ Entretanto, como o número de situações no dia a dia do laboratório não é trivial, a identificação e quantificação de todas as fontes de incerteza fizeram com que os químicos analíticos relutassem em adotar esta abordagem. Em contra partida, o Comitê de Métodos Analíticos (AMC) da Sociedade Real de Química retomou a proposta de Wernimont, introduzindo a abordagem top-down baseada em estudos interlaboratoriais. ${ }^{2}$ Logo após, o Comitê Nórdico para Análises de Alimentos (NMKL) sugeriu uma abordagem similar, baseada somente em dados provenientes de estudos intralaboratoriais. ${ }^{6}$ Os dados necessários para a abordagem topdown, ou ao menos uma parte deles, já estão geralmente disponíveis na validação do método.

A discussão na avaliação da incerteza de medição influenciou também o ISO/IEC 17025 e os laboratórios que requisitam a acreditação. ${ }^{7}$ A seção desta norma sobre incerteza cita que "a estimativa

*e-mail: elciooliveira@petrobras.com.br razoável deve estar baseada no conhecimento do desempenho do método e no escopo da medição, e deve fazer uso, por exemplo, de experiência e dados de validação anteriores". A norma faz referência explícita ao GUM, ${ }^{3}$ mas também ao padrão de ISO de exatidão dos resultados de medição. ${ }^{8} \mathrm{Um}$ dos exemplos da segunda edição do Guia da EURACHEM, ${ }^{9}$ bem como a IUPAC,${ }^{10}$ sugerem usar dados de validação na estimativa da incerteza, também. Este último se refere não somente aos estudos interlaboratoriais, ${ }^{11}$ mas menciona também a possibilidade para incluir a informação dos testes de robustez. ${ }^{12,13}$ Os testes de robustez verificam através de uma abordagem intralaboratorial se o método é suscetível a pequenas modificações deliberadamente introduzidas aos seus parâmetros, que podem ocorrer quando este é transferido entre laboratórios (ou tempo, ou analistas, ou instrumentos). Se as modificações introduzidas forem escolhidas adequadamente, um teste de robustez pode ser considerado como uma simulação de um estudo interlaboratorial. Uma estimativa intralaboratorial do desvio padrão da reprodutibilidade obtida nesta maneira pode ser usada como estimativa da incerteza. Barwick e Ellison propõem uma abordagem diferente para integrar os dados de robustez na avaliação da incerteza. ${ }^{14}$ Esta abordagem utiliza dados de robustez como um suplemento aos dados de precisão e recuperação. ${ }^{15,16}$ As fontes de incerteza que não são cobertas pela recuperação são obtidas nos testes de robustez.

Os métodos fuzzy foram apresentados por Zadeh em 1965. ${ }^{17}$ Ele descreveu um caminho para se avaliar afirmativas vagas ou observações incertas. Caso as variáveis de um processo possam ser consideradas como $f u z z y,{ }^{18}$ é possível se utilizar esta abordagem para avaliar a incerteza de medição.

Em 2002, um grupo de pesquisadores europeus elaborou um trabalho, no qual comparam algumas abordagens para estimar a incerteza no ensaio de cromatografia líquida da tilosina para uso veterinário. ${ }^{19}$ Neste estudo, a precisão do plano interlaboratorial é limitada à condição de repetitividade em seus experimentos e não avalia a condição de normalidade dos dados. A etapa de validação é realizada com um método padrão diferente do proposto, além de não abordarem a aritmética fuzzy. Os autores daquele artigo comparam desvio-padrão ao 
invés de incerteza expandida, não compensando o efeito dos diferentes graus de liberdade, pelo uso do fator de abrangência.

Neste trabalho, um teste de robustez da cromatografia gasosa do gás natural é executado no decorrer do plano interlaboratorial. A partir destes dados, é possível se fazer um estudo comparativo das diferentes abordagens. A avaliação da incerteza considera somente o processo analítico, não levando em consideração a incerteza devida à amostragem.

\section{PARTE EXPERIMENTAL}

\section{Amostra}

A amostra utilizada foi o gás natural (gás combustível processado), que consiste em uma mistura de hidrocarbonetos, principalmente metano, etano, propano e hidrocarbonetos de maior massa molecular que se encontram em quantidades menores. Apresenta normalmente gases inertes, tais como nitrogênio, dióxido de carbono, bem como traços de outros constituintes. Os componentes analisados foram: metano, etano, propano, isobutano, butano, isopentano, pentano, dióxido de carbono e nitrogênio.

\section{Procedimento cromatográfico}

Geralmente, em casos em que a composição da corrente de gás natural é estável e conhecida, como nas indústrias brasileiras de produção de energia, é comum usar uma calibração com único ponto ao invés da calibração com múltiplos pontos. ${ }^{20}$

As metodologias exigidas pela Portaria ANP (Agência Nacional de Petróleo) $\mathrm{N}^{\mathrm{o}} 104,{ }^{21}$ para a análise da composição do gás natural são: ISO $6974^{22}$ e ASTM D $1945 .{ }^{23}$

As condições mais utilizadas na análise por cromatografia gasosa (CG) com padronização externa são mostradas na Tabela 1.

Tabela 1. Parâmetros para a análise de CG para o gás natural, com padronização externa
Coluna cromatográfica

Gás de arraste

Vazão do gás de arraste

Detector

Sistema de injeção

Temperatura do bloco (coluna, detector e filamento)
Porapak, chromosorb ou peneira molecular

Hidrogênio ou hélio

30 a $60 \mathrm{~mL} / \mathrm{min}$

Condutividade térmica (DCT) ou ionização de chama (FID)

60 a $90{ }^{\circ} \mathrm{C}$
Automático

\section{Estudo interlaboratorial}

O plano interlaboratorial é uma forma de avaliar o desempenho de um método, nas condições normais de trabalho, em diversos laboratórios, através de ensaio de amostras homogêneas e estáveis, preparadas por um laboratório provedor.

Neste estudo, somente um dos laboratórios participantes do plano interlaboratorial executou o teste de robustez. Um único cilindro contendo a amostra de gás natural foi analisado por 13 laboratórios. A amostra foi analisada em replicatas $(2 \leq n \leq 10)$ sob condições de precisão intermediária ao longo do tempo e nas mesmas condições descritas na Tabela $1 .{ }^{24} \mathrm{~A}$ composição de gás natural desta amostra, baseada na norma ISO $6142,{ }^{25}$ encontra-se na Tabela 2.
Tabela 2. Composição de gás natural da amostra analisada

\begin{tabular}{llcc}
\hline Componente & & \multicolumn{2}{c}{$\%$ mol/mol } \\
\cline { 3 - 4 } & & Concentração & Incerteza expandida \\
\hline Etano & $\mathrm{C} 2$ & 7,010 & $\pm 0,040$ \\
Propano & $\mathrm{C} 3$ & 2,000 & $\pm 0,010$ \\
Isobutano & $\mathrm{iC} 4$ & 0,248 & $\pm 0,003$ \\
Butano & $\mathrm{C} 4$ & 0,292 & $\pm 0,003$ \\
Isopentano & $\mathrm{iC} 5$ & 0,090 & $\pm 0,001$ \\
Pentano & $\mathrm{C} 5$ & 0,080 & $\pm 0,001$ \\
Dióxido de carbono & $\mathrm{CO}$ & 0,694 & $\pm 0,007$ \\
Nitrogênio & $\mathrm{N}_{2}$ & 0,915 & $\pm 0,009$ \\
Metano & $\mathrm{C} 1$ & 88,670 & $\pm 0,190$ \\
\hline
\end{tabular}

\section{Teste de robustez}

A robustez de um método de ensaio mede a sensibilidade que este apresenta face a pequenas variações. Um método é dito robusto quando pequenas variações que possam ocorrer quando esse está sendo executado, não influenciam significativamente a resposta mensurada.

A Tabela 3 mostra os fatores e os níveis examinados, que foram considerados como potenciais fontes de incerteza, baseados na literatura. ${ }^{26,27}$ As modificações apresentadas foram escolhidas na faixa que pode ser usualmente esperada quando há transferência do método entre laboratórios diferentes. $\mathrm{O}$ teste de robustez foi executado na mesma amostra do plano interlaboratorial, em 3 dias consecutivos: $1^{\circ}$ dia, $2^{\circ}$ dia e $3^{\circ}$ dia.

A amostra e o material de referência certificado (MRC) foram analisados ao longo do teste de robustez. A partir destes dados, uma estimativa da precisão intermediária ao longo do tempo pode ser obtida.

\section{DIFERENTES ABORDAGENS DE INCERTEZA}

\section{Abordagem GUM}

A avaliação da incerteza segundo o GUM (também conhecida como abordagem bottom-up) consiste na identificação e quantificação das fontes relevantes de incerteza, ${ }^{28}$ seguida da combinação de suas estimativas individuais. Quando expressa como um desvio padrão, um componente da incerteza é conhecido como incerteza padrão, $u(x)$. Se existe correlação entre alguns componentes, deve-se calcular a correlação ou covariância.

Para um resultado de medição $x$, a incerteza padrão combinada, $u_{c}(x)$ é uma estimativa do desvio padrão combinado, e é igual à raiz quadrada positiva da variância total obtida pela combinação de todos os componentes de incerteza avaliados, usando a lei da propagação de incerteza, que se baseia na expansão da Série de Taylor. ${ }^{3}$

Para muitos propósitos na Química Analítica, ${ }^{5}$ a incerteza expandida, $U(x)$ é usada. A incerteza expandida provém do intervalo dentro do qual o valor do mensurando é acreditado estar com um nível particular de confiança. $U(x)$ é obtido pela multiplicação de $u_{c}(x)$, a incerteza padrão combinada, por um fator de abrangência $k$. A escolha do fator $k$ é baseada no nível de confiança desejado.

O modelo matemático utilizado para calcular a fração molar não-normalizada do componente $i$ do gás natural por cromatografia gasosa é:

$\stackrel{*}{x}_{i}=\frac{X_{M R C, i}}{\hat{R}_{M R C, i}} \hat{R}_{i}$ 
Tabela 3. Fatores e níveis examinados no teste de robustez

\begin{tabular}{|c|c|c|c|c|c|c|c|c|c|c|c|}
\hline \multicolumn{4}{|c|}{$1^{\circ} \mathrm{dia}$} & \multicolumn{4}{|c|}{$2^{\circ} \mathrm{dia}$} & \multicolumn{4}{|c|}{$3^{\circ} \mathrm{dia}$} \\
\hline \multicolumn{2}{|c|}{ Temperatura } & \multicolumn{2}{|c|}{ Vazão } & \multicolumn{2}{|c|}{ Temperatura } & \multicolumn{2}{|c|}{ Vazão } & \multicolumn{2}{|c|}{ Temperatura } & \multicolumn{2}{|c|}{ Vazão } \\
\hline Valor $\left({ }^{\circ} \mathrm{C}\right)$ & Nível & $\begin{array}{c}\text { Valor (mL/ } \\
\text { min) }\end{array}$ & Nível & Valor $\left({ }^{\circ} \mathrm{C}\right)$ & Nível & $\begin{array}{c}\text { Valor (mL/ } \\
\text { min) }\end{array}$ & Nível & Valor $\left({ }^{\circ} \mathrm{C}\right)$ & Nível & $\begin{array}{c}\text { Valor (mL/ } \\
\text { min) }\end{array}$ & Nível \\
\hline 59 & -1 & 34 & -1 & 61 & +1 & 34 & -1 & 60 & 0 & 40 & 0 \\
\hline 59 & -1 & 46 & +1 & 61 & +1 & 46 & +1 & 60 & 0 & 40 & 0 \\
\hline 61 & +1 & 34 & -1 & 60 & 0 & 40 & 0 & 60 & 0 & 40 & 0 \\
\hline 61 & +1 & 46 & +1 & 60 & 0 & 40 & 0 & 59 & -1 & 34 & -1 \\
\hline 60 & 0 & 40 & 0 & 60 & 0 & 40 & 0 & 59 & -1 & 46 & +1 \\
\hline 60 & 0 & 40 & 0 & 59 & -1 & 34 & -1 & 61 & +1 & 34 & -1 \\
\hline 60 & 0 & 40 & 0 & 59 & -1 & 46 & +1 & 61 & +1 & 46 & +1 \\
\hline
\end{tabular}

onde: $x_{i}$ é a fração não-normalizada do componente $i ; x_{\mathrm{MRC.i}}$ é a fração molar do componente $i$ do MRC, disponível no certificado; $\mathrm{R}_{\mathrm{MRC}, \mathrm{i}}$ a área do pico do componente $i$ do MRC, analisada; $R_{i}$ é a área do pico do componente $i$ da amostra, analisada.

A incerteza da fração molar não-normalizada do componente $i$, aplicando o GUM, na Equação 1, ao considerarmos as grandezas não correlacionadas entre si, é: ${ }^{29}$

$\left(\begin{array}{c}u_{*} \\ \frac{x_{i}}{*} \\ x_{i}\end{array}\right)^{2}=\left(\frac{u_{x_{M R C, i}}}{x_{M R C, i}}\right)^{2}+\left(\frac{u_{\hat{R}_{M R C, i}}}{\hat{R}_{M R C, i}}\right)^{2}+\left(\frac{u_{\hat{R}_{i}}}{\hat{R}_{i}}\right)^{2}$

onde: $\mathrm{u}_{\mathrm{xi}}$ é a incerteza padrão da fração molar não-normalizada do componente $i$; $\mathrm{u}_{\mathrm{x}_{\text {MRC }}}$ é a incerteza padrão da fração molar do componente $i$ do MRC, disponível no certificado; $\mathrm{u}_{\hat{\mathrm{R}}_{\text {Мвсі }}}$ é a incerteza padrão da área do pico do componente $i$ do MRC, analisada; $\mathrm{u}_{\hat{R}_{i}}$ é a incerteza padrão da área do pico do componente $i$ da amostra, analisada.

O modelo matemático utilizado para calcular a fração molar normalizada do componente $i$ da amostra, é:

$X_{i}=\frac{\boldsymbol{X}_{i}}{\sum_{w=1}^{q} \boldsymbol{X}_{w}^{*}}$

onde: $x_{i}$ é a fração molar normalizada do componente $i ; x_{w}$ é a fração molar não-normalizada de cada componente $i$.

A incerteza da fração molar normalizada do componente $i$ da amostra é:

$$
u_{x_{i}}=x_{i} \sqrt{\frac{1-2 x_{i}^{*}}{x_{i}{ }^{2}} u_{* i^{2}}+\sum_{w=1}^{q} u_{x w^{2}}}
$$

\section{A abordagem top-down: avaliação da incerteza a partir dos dados de reprodutibilidade}

Este trabalho avalia a precisão interlaboratorial baseado no padrão ISO 5725-2 modificado. $^{8}$ A etapa de modificação da metodologia baseia-se na exclusão de valores aberrantes, onde são descartados valores com escore-z maiores que 3 (três). ${ }^{30} \mathrm{O}$ escore-z indica o quanto acima ou abaixo da média um escore está em termos de unidades padronizadas de desvio-padrão. Através da abordagem de análise de variância (ANOVA) ${ }^{31}$ diferentes avaliações de variância são obtidas a partir de estudos interlaboratoriais. $\mathrm{Na}$ abordagem top-down, o desvio padrão da reprodutibilidade obtido, $s_{R}$, é considerado como a incerteza padrão de um resultado de medição, quando os dados apresentam distribuição normal. ${ }^{3}$
Se várias amostras, por exemplo, a várias concentrações ou diferentes matrizes, são incluídas na avaliação de incerteza, a incerteza padrão de um resultado de medição pode ser obtida pelo agrupamento de desvios-padrão relativos de reprodutibilidade, desde que os desvios-padrão relativos para amostras diferentes sejam comparáveis. Se este não for o caso, a incerteza é individualmente determinada para matrizes ou faixas de concentração diferentes. ${ }^{32}$

\section{A abordagem de Barwick e Ellison: combinação de dados da validação}

Barwick e Ellison propõem combinar três diferentes elementos do método de validação a fim de avaliar a incerteza: ${ }^{14}$ precisão, recuperação e robustez. ${ }^{33}$ As estimativas da precisão intermediária relativas a diferentes tempo e/ou operador são consideradas.

Com o objetivo de cobrir as incertezas relativas ao erro sistemático do método (tendência), estimativas de recuperação também devem ser consideradas.

Barwick e Ellison descrevem várias possibilidades para estimar a incerteza relativa à recuperação, incluindo a análise de materiais de referência certificados (MRC), fortificação (spiking) e comparação com um método de referência. Dentre estas alternativas aqui consideradas, a utilização de MRC foi aplicada neste trabalho. A recuperação média do método é dada por:

$\bar{R}_{m}=\bar{C}_{\text {método }} / C_{\text {padrão }}$

onde: $\bar{C}_{\text {método }}$ é a média os resultados obtidos usando o método a ser validado e $C_{\text {padrão }}$ ó resultado proveniente do certificado do material de referência. A incerteza na recuperação, $u(\bar{R} m)$, é dada por: ${ }^{34}$

$$
u\left(\bar{R}_{m}\right)=\bar{R}_{m} \sqrt{\frac{s_{\text {método }}^{2}}{n \bar{C}_{\text {método }}^{2}}+\left(\frac{u\left(C_{\text {padrão }}\right)}{C_{\text {padrão }}}\right)^{z}}
$$

onde: $s_{\text {método }}$ é o desvio padrão de resultados obtidos usando o método, $n$ é número de replicatas e $u\left(C_{\text {padrão }}\right)$ é a incerteza padrão associada ao MRC. A incerteza padrão do MRC é utilizada como desvio padrão. Se a recuperação é significantemente diferente de 1, devemos usar este fator para corrigir o resultado de medição.

Tanto a precisão quanto a recuperação são avaliadas dentro de um único laboratório, o que não considera fontes de incerteza devidas a condições diferentes como, por exemplo, aquelas provenientes do uso de laboratórios diferentes. Como o teste de robustez examina as modificações dos parâmetros do método esperadas na transferência entre laboratórios, os dados de robustez devem ser considerados adicionalmente na avaliação da incerteza. Embora 
Barwick e Ellison recomendem que a utilização da estimativa de precisão seja avaliada sob um curto período de tempo; ${ }^{14}$ aqui, o desvio padrão da precisão intermediária em função do tempo, $S_{/(T)}$ é utilizado, pois a repetitividade geralmente conduz a uma super estimativa do número de efeitos significativos. ${ }^{35}$ Para estes fatores que não produzem efeitos significativos, a incerteza leva em consideração situações reais nas quais a variabilidade de alguns fatores possa ser menor do que o teste de robustez. Consequentemente, a incerteza padrão corresponde ao desvio padrão que deve ser esperado com a variação real.

Para obter a incerteza padrão combinada $u_{c}(x)$, as estimativas da incerteza para diferentes fatores $p, q, r, \ldots$ do teste de robustez são combinadas com as estimativas para recuperação e precisão intermediária:

$$
\frac{u_{c}(x)}{x}=\sqrt{\left(\frac{u\left(\bar{R}_{m}\right)}{\bar{R}_{m}}\right)^{2}+\left(\frac{s_{l(T)}}{\bar{x}}\right)^{2}+\left(\frac{u(p)}{p}\right)^{2}+\left(\frac{u(q)}{q}\right)^{2}+\left(\frac{u(r)}{r}\right)^{2}+\ldots}
$$

\section{Estudo de variabilidade: somente a partir dos dados de} robustez

Os testes de robustez podem ser considerados como sendo simulações intralaboratoriais de estudos interlaboratoriais, se as modificações apresentadas forem adequadamente escolhidas. Isto permite uma posterior estimativa da incerteza do resultado de medição. Em contraste com a abordagem proposta por Barwick e Ellison, que não considera separadamente os fatores diferentes examinados no teste de robustez, esta abordagem pondera as variações provenientes do planejamento de experimentos. Deste modo, os dados de precisão provenientes dos testes de robustez são utilizados da mesma maneira que as avaliações de incerteza a partir dos dados de reprodutibilidade, após a constatação da normalidade dos dados. ${ }^{3}$

\section{Aritmética fuzzy}

Um número fuzzy é definido como uma função de pertinência em torno de um valor considerado mais provável, podendo ter diversas representações possíveis. ${ }^{36} \mathrm{~A}$ forma do número fuzzy deve ser escolhida tentando se aproximar do comportamento da variável medida, tendo como uma das representações mais simples encontrada na literatura, a triangular.

Os termos $\alpha$ e $\beta$ foram obtidos de forma a se ter os limites de certeza da medição com valores de pertinência que variem entre $0 \leq \mathrm{p} \leq$ $1,{ }^{37}$ abrangendo todo o domínio da variável. Os valores de pertinência podem variar nesta faixa, desde que estes valores utilizados na etapa de fuzzificação, sejam os mesmos utilizados na defuzzificação.
Com o uso da aritmética fuzzy, obtêm-se diretamente os resultados de cálculos com as respectivas incertezas, por tratar as variáveis não mais como um número que representa de forma determinística a grandeza medida, mas como uma função de pertinência em torno de um valor mais provável.

\section{RESULTADOS E DISCUSSÃO}

\section{Abordagem GUM}

Os valores de incerteza expandida encontram-se na Tabela 4, sendo que os da esquerda representam a incerteza da abordagem GUM dos componentes normalizados e os da direita, não-normalizado.

É possível se observar que o algoritmo de normalização gera os seguintes efeitos nesta abordagem: aumento da incerteza do componente, à medida que aumenta a incerteza do MRC, com exceção do metano; minimização considerável da incerteza do componente com mais de $50 \%$ na amostra.

Esta abordagem permite estratificar detalhadamente as fontes de incerteza, Figura 1. Com exceção do C2 e C3, a maior contribuição é proveniente da incerteza do MRC.

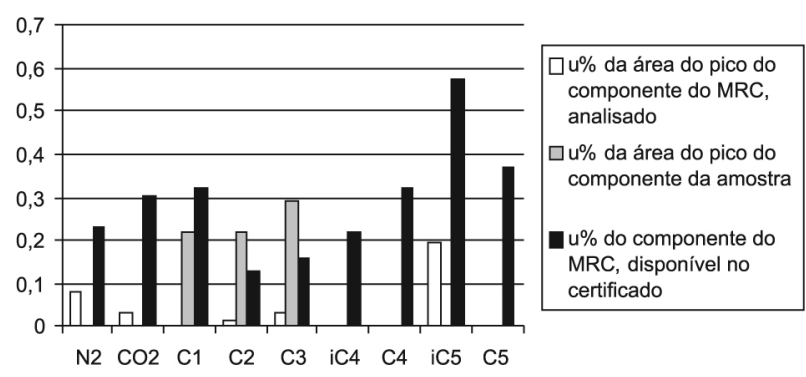

Figura 1. Contribuição de cada fonte de incerteza

\section{A abordagem top-down: avaliação da incerteza a partir dos dados de reprodutibilidade}

A avaliação de incerteza baseada no estudo interlaboratorial para os componentes do gás natural está resumida na Tabela 4. A incerteza de cada componente é proveniente de todos os resultados de diferentes laboratórios, logo, corresponde ao desvio padrão em termos de reprodutibilidade.

A fim de avaliar a normalidade dos dados, utilizamos o teste de Kolmogorov-Smirnov. ${ }^{31}$ Entretanto, devido ao comportamento não-normal dos dados, o desvio não padrão representa bem a dispersão dos dados.

Tabela 4. Valores de incerteza expandida

\begin{tabular}{|c|c|c|c|c|c|c|c|}
\hline \multirow[b]{2}{*}{ Componente } & \multirow{2}{*}{$\begin{array}{c}\text { Composição } \\
(\%)\end{array}$} & \multicolumn{6}{|c|}{ Incerteza expandida relativa } \\
\hline & & MRC & GUM & Top-down & $\begin{array}{l}\text { Barwick e } \\
\text { Ellison }\end{array}$ & Variabilidade & Fuzzy \\
\hline $\mathrm{N}_{2}$ & 0,90 & 0,40 & $1,4 / 0,81$ & 8,6 & 1,6 & 0,92 & 1,5 \\
\hline $\mathrm{CO}_{2}$ & 0,64 & 0,53 & $1,5 / 1,0$ & 4,6 & 1,7 & 0,95 & 1,6 \\
\hline $\mathrm{C} 1$ & 88,93 & 0,56 & $0,21 / 1,8$ & 0,53 & 1,7 & 0,12 & 2,0 \\
\hline $\mathrm{C} 2$ & 6,87 & 0,22 & $1,4 / 0,81$ & 4,1 & 1,6 & 0,25 & 1,6 \\
\hline $\mathrm{C} 3$ & 1,95 & 0,27 & $1,4 / 0,96$ & 4,0 & 1,6 & 6,5 & 1,6 \\
\hline $\mathrm{iC} 4$ & 0,24 & 0,38 & $1,4 / 0,73$ & 4,6 & 1,6 & 2,8 & 1,3 \\
\hline $\mathrm{C} 4$ & 0,30 & 0,56 & $1,6 / 1,1$ & 6,6 & 1,7 & 2,6 & 1,5 \\
\hline $\mathrm{iC} 5$ & 0,09 & 1,0 & $2,3 / 2,0$ & 9,9 & 2,0 & 10 & 2,4 \\
\hline $\mathrm{C} 5$ & 0,08 & 0,64 & $1,7 / 1,2$ & 6,8 & 1,7 & 5,7 & 1,6 \\
\hline
\end{tabular}


Baseado em resultados oriundos da Figura 1 que, de maneira geral, a maior fonte de incerteza é devido ao MRC, concluímos que os valores de incerteza, nesta abordagem são bem mais elevados do que a abordagem GUM; pois, cada laboratório calibra o seu cromatógrafo com MRC com incertezas distintas.

\section{A abordagem de Barwick e Ellison: combinação de dados da validação}

Enquanto a abordagem top-down somente considera estimativas de precisão a partir de um estudo interlaboratorial, aqui a precisão intermediária estimada ao longo do tempo é considerada, e é complementada pelas incertezas estimadas a partir da avaliação de robustez e recuperação. Os valores de robustez utilizados no teste são: $\pm 1{ }^{\circ} \mathrm{C}$ na temperatura do bloco (coluna, forno, filamento e detector); $\pm 6 \mathrm{~mL} / \mathrm{min}$ na vazão do gás de arraste. Enquanto que os valores reais de robustez são: $\pm 0,009{ }^{\circ} \mathrm{C}$ na temperatura do bloco (coluna, forno, filamento e detector); $\pm 0,010 \mathrm{~mL} / \mathrm{min}$ na vazão do gás de arraste.

É possível se observar, Tabela 4, que os valores de incerteza praticamente não variaram. Este comportamento pode ser atribuído a grande contribuição dos fatores de robustez - vazão do gás de arraste e temperatura do bloco - que é constante para todos os componentes, Figura 2.

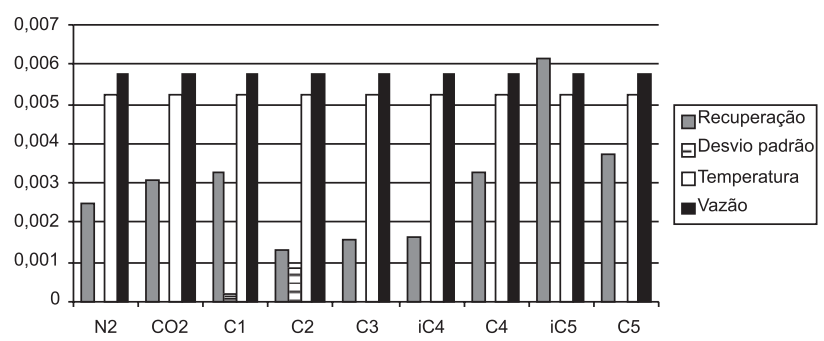

Figura 2. Contribuição de cada componente da incerteza na amostra

\section{Estudo de variabilidade: somente a partir dos dados de robustez}

Para esta abordagem, o significado dos efeitos no teste de robustez foi determinado anteriormente.

As estimativas de incertezas obtidas a partir da variância de planejamento de experimentos do teste de robustez são mostradas na Tabela 4. A fim de avaliar a normalidade dos dados, utilizamos novamente o teste de Kolmogorov-Smirnov. ${ }^{31}$ Entretanto, devido ao comportamento não-normal dos dados, o desvio não padrão representa bem a dispersão dos dados.

\section{Aritmética fuzzy}

A fuzzificação dos dados de entrada se realiza em duas etapas, onde são levados em consideração: os valores de fração molar e seus respectivos desvios-padrão de cada componente do MRC, encontrados no certificado de calibração, a área dos picos e seus respectivos desvios-padrão de cada componente do MRC analisado e a área dos picos e seus respectivos desvios-padrão do componente analisado da amostra, conforme Equação 1; os valores da fração molar nãonormalizada e seus respectivos desvios-padrão de cada componente da amostra e o somatório e seus respectivos desvios-padrão das frações molares não-normalizadas, conforme Equação 3.

Os resultados provenientes da aritmética fuzzy geraram as incertezas expandidas relativas apresentadas na Tabela 4.

\section{Comparação das diferentes abordagens}

Os resultados obtidos pela abordagem top-down e pelo estudo de variabilidade não são comparáveis às demais abordagens, pela não normalidade dos dados.

A abordagem top-down resulta, de maneira geral, de estimativas maiores da incerteza do que os outros métodos considerados. Isto não é ilógico, porque esta abordagem considera explicitamente a incerteza devida à transferência entre os laboratórios, influência esta que pode estar subestimada nas outras abordagens. Também acreditamos que isto possa acontecer, pois, o MRC é a contribuição de maior peso e a sua incerteza pode variar muito de laboratório para laboratório. Por exemplo, foram encontrados valores de incerteza do MRC para o iC5 de $\pm 0,32$ até \pm $1,2 \%$, dentre os laboratórios participantes do plano interlaboratorial.

Também foi observado que as diferentes condições dos parâmetros cromatográficos não foram levadas em consideração explicitamente nas abordagens GUM e fuzzy, como grandezas de entrada. No entanto, esta variabilidade se refletiu na grandeza de saída, a ponto de fazer com que estas abordagens fossem comparáveis com a de Barwick e Ellison, que explicita estas fontes.

Como é possível observar, há uma grande compatibilidade entre as abordagens GUM, Barwick e Ellison e fuzzy, exceto para o componente $\mathrm{C} 1$. Estas incertezas apresentam valores comparáveis ao estudo do CCQM. ${ }^{38}$ Quanto ao C1, esta diferença pode ser atribuída ao efeito do algoritmo da normalização, que minimiza a incerteza para este componente que se apresenta em mais de $50 \%$ na composição do gás natural, conforme constatado em estudo anterior, por Brown. ${ }^{39}$

\section{CONCLUSÃO}

Demonstrou-se que as abordagens GUM, validação e fuzzy conduzem a estimativas de incerteza comparáveis; o que não acontece com a abordagem top-down e o estudo da variabilidade pela ausência de normalidade dos dados. Observamos que, de maneira geral, as estimativas da incerteza relativa avaliadas pela abordagem GUM são um pouco menores do que as estimativas das outras duas abordagens, Barwick e Ellison e fuzzy. Acredita-se que isto pode ser devido ao fato de algumas fontes da incerteza terem sido negligenciadas, que as fontes consideradas tenham sido subestimadas ou, ainda, a não consideração da covariância entre os componentes. As abordagens de acordo com Barwick e Ellison e a que envolve somente dados de robustez podem ser vantajosas sobre a abordagem top-down, porque têm menor custo uma vez que o número das experiências a ser executado, além dos experimentos de validação já existentes, é normalmente mais baixo. Em contrapartida, somente as abordagens do GUM, de acordo com Barwick e Ellison e fuzzy fornecem informação sobre as contribuições dos diferentes parâmetros do método à incerteza padrão combinada. Se a incerteza de um método for avaliada pela abordagem da variabilidade é imprescindível se certificar de que o teste da robustez examina certamente aquelas modificações que são prováveis ocorrer em transferência entre laboratórios.

\section{AGRADECIMENTOS}

Aos químicos da PETROBRAS M. M Gomes e A. C. dos Santos pela ajuda na aquisição dos dados de robustez e do plano interlaboratorial.

\section{REFERÊNCIAS}

1. International Standard Organization; Statistics-Vocabulary and symbols - Part 1: Probability and general statistical terms, Geneva, Switzerland, 1993. 
2. Analytical Methods Committee; Analyst 1995, 120, 2303.

3. International Standard Organization; Guide to the Expression of Uncertainty in Measurement, $1^{\text {st }}$ ed., Geneva, Switzerland, 1993.

4. Wernimont, G. T.; Use of Statistics to Develop and Evaluate Analytical Methods, AOAC: Arlington, 1985.

5. EURACHEM; Quantifying uncertainty in analytical measurement, $1^{\text {st }}$ ed., Helsinki, 1995.

6. http://www.nmkl.org/Engelsk/index.htm, Acessada em Fevereiro 2009 .

7. International Standard Organization; General Requirements for the Competence of Testing and Calibration Laboratories, ISO/IEC 17025, 2005.

8. International Standard Organization; Precision of Test Methods, ISO 5725-2, 1994.

9. EURACHEM; Quantifying uncertainty in analytical measurement. $2^{\text {nd }}$ ed., Helsinki, 2000.

10. Report on the FAO, IAEA, AOAC INT/IUPAC; International Workshop on Principles and Practices of Method Validation, Budapest, 1999 (IUPAC Projects 5/97/8 and 5/2/99).

11. Cox, M. G.; Harris, P. M.; Meas. Sci. Technol. 2006, 17, 533.

12. Pavese, F.; Measurement 2006, 39, 779.

13. Willink, R.; Metrologia 2008, 45, 442.

14. Barwick, V. J.; Ellison, S. L. R.; VAM Project 3.2.1, Development and Harmonisation of Measurement Uncertainty Principles. Part d. Protocol for Uncertainty Evaluation from Validation Data, Version 5.1, January 2000.

15. Vanatta, L. E.; Coleman, D. E.; J. Chromatogr., A 2007, 1158, 47.

16. Lissinger, T. P. J.; Trends Anal. Chem. 2008, 27, 916.

17. Zadeh, L. A.; Inform. Control. 1965, 8, 338.

18. Ferrero, A. A.; Salicone, S. S.; IEEE Trans. Instrum. Meas., (2008), doi: 10.1109/TIM.2008.928873.

19. Hund, E.; Massart, D. L.; Smeyers-Verbeke, J.; Anal. Chim. Acta 2003, $480,39$.

20. Leito, S.; Mölder, K.; Künnapas, A.; Herodes, K.; Leito, I.; J. Chromatogr., A 2006, 1121, 55.
21. Agência Nacional do Petróleo (ANP); Portaria $N^{\circ} 104$, de 8 de julho de 2002 - Estabelece a especificação do gás natural, de origem nacional ou importada, a ser comercializado em todo território nacional.

22. International Standard Organization; Natural Gas - Determination of composition with defined uncertainty by gas chromatography, ISO 6974, Parts 1 to 5, 2001.

23. American Society for Testing and Materials; Standard Test Method for Analysis of Natural Gas by Gas Chromatography, ASTM D 1945, 2001.

24. Meyer, V. R., J. Chromatogr., A 2007, 1158, 15.

25. International Standard Organization; Gas Analysis - Preparation of Calibration Gas Mixtures - Gravimetric Method, ISO 6142, 2001.

26. Barros Neto, B.; Scarminio, I. S.; Bruns, R. E.; Como Fazer Experimentos - Pesquisa e Desenvolvimento na Ciência e na Indústria, Ed. da Unicamp: Campinas, 2001.

27. Barwick, V. J.; J. Chromatogr., A 1999, 849, 13.

28. Bich, W.; Cox, M. G.; Harris, P. M.; Metrologia 2006, 43, S161.

29. Wang, D.; Wang, S.; Zhao, S.; J. Test. Eval. 2005, 33, 3.

30. International Standard Organization; Proficiency Testing by Interlaboratory Comparisons - Part 1: Development and Operation of Laboratory Proficiency Testing Schemes, ISO Guide 43-1, 1997.

31. Miller, J. N.; Miller, J. C.; Statistics and Chemometrics for Analytical Chemistry, $4^{\text {th }}$ ed., Prentice Hall: United Kingdom, 2000.

32. Thompson, M.; Ellison, S. L. R.; Accred. Qual. Assur. 2005, 10, 82.

33. Dias, M. G.; Camões, M. F. G. F. C.; Oliveira, L.; Food Chem. 2008, 109, 815 .

34. Gluschke, M.; Wellmitz, J.; Lepom, P.; Accred. Qual. Assur. 2005, 10, 107.

35. Heyden, Y. V.; Luypaert, K.; Hartmann, C.; Massart, D. L.; Hoogmartens, J.; De Beer, J.; Anal. Chim. Acta 1995, 312, 245.

36. Pedrycz, W.; Gomide, F.; An introduction to fuzzy sets: Analysis and design, MIT Press, 1998.

37. Hayward, G.; Davidson, V.; Analyst 2003, 128, 1304.

38. Veen, A. M. H.; Final Report International Comparison CCQM K23ac - Natural gas types I and III. Metrologia 2007, 08001, doi:10.1088/0026-1394/44/1A/08001.

39. Brown, A. S.; J. Chromatogr., A 2004, 1040, 215. 